\title{
SKETCH INPUT OF 3D MODELS Current Directions
}

\author{
Peter Varley and Pedro Company \\ Universitat Jaume I, Dept. of Mechanical Engineering and Construction, E-12071, Castellon, Spain \\ varley@emc.uji.es,pcompany@emc.uji.es
}

Keywords: $\quad$ Sketch Input, Solid Models, Computer-Aided Design, Natural Line Drawings, Wireframe Drawings.

\begin{abstract}
In the last few years, there has been considerable interest in sketch input of 3D solid models. This paper summarises recent developments and discusses the directions these developments are taking. We consider three developments in particular: the move away from line labelling as a technique in recognition of the problem posed by extended vertices; the increasing use of symmetry detection as a tool for reconstruction; and progress towards interpretation of drawings depicting curved objects.
\end{abstract}

\section{INTRODUCTION}

\subsection{Objective}

Our overall goal is to create a $3 \mathrm{D}$ solid model automatically from a single 2D drawing. A tool which could quickly interpret line drawings of engineering objects as boundary representation CAD models would be of significant benefit in the process of engineering design. It would enable designers to spend more time on the creative aspects of their job and less on the routine aspects, it would reduce time spent correcting mistakes by allowing instant visualisation, and the simpler "what you draw is what you imagine” interface will be less distracting than an array of menus and icons.

\subsection{Terminology}

A drawing depicts an object. The junctions, lines and regions of the drawing often, but not always, correspond to the vertices, edges and faces of the object.

A drawing is a natural line drawing if it depicts only those parts of the object visible from some chosen viewpoint. It is a wireframe drawing if it depicts all vertices and edges of the object.

A vertex is trihedral if exactly three edges meet at it. An object is trihedral if all of its vertices are trihedral.

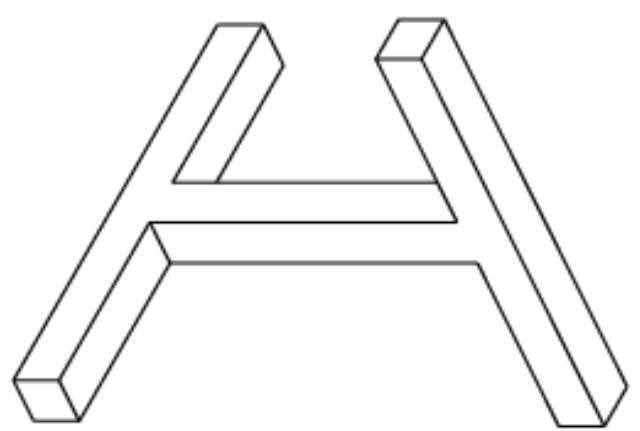

Figure 1: Natural Line Drawing.

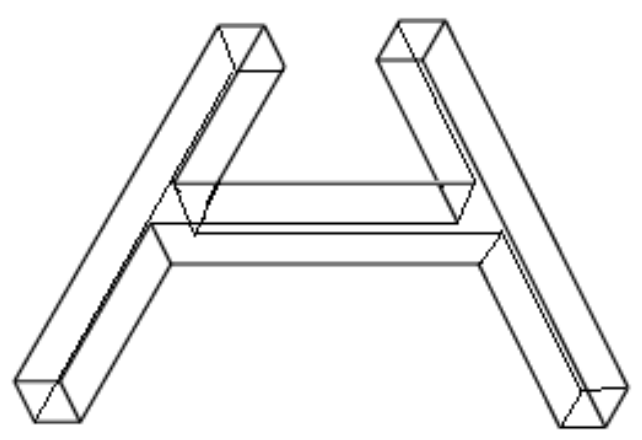

Figure 2: Wireframe Drawing. 


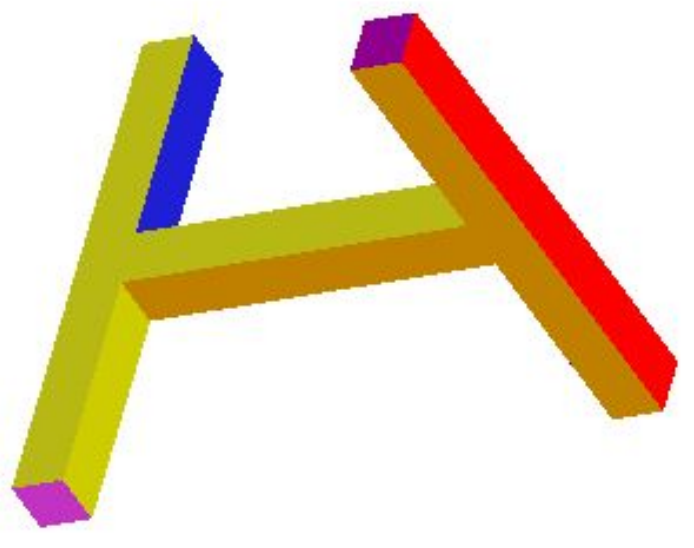

Figure 3: Object.

In engineering, our main interest is in solid objects, the faces of which bound a single continuous finite volume. A solid object is a polyhedron if all of its faces are planar. A polyhedron is a normalon if all of its edges and face normals are aligned with one of three mutually orthogonal axes, or a quasi-normalon if all of its vertices terminate at least one edge aligned with one of the three mutually orthogonal axes.

An object or drawing is described by its topology (discrete data such as vertex/edge connectivity) and geometry (continuous data such as vertex coordinates and edge lengths). A drawing is from a general viewpoint if no small change in the viewpoint changes the topology of the drawing. We assume that all drawings are from general viewpoints.

\subsection{Structure of Paper}

This paper describes recent progress in interpreting both natural line drawings and wireframe drawings. There are some problems unique to one or the other (for example, the question of what is around the back of the object is unique to natural line drawings), but there are also many problems, notably that of determining design intent, which are common to both.

Section 2 describes our baseline, the state of the art as presented at the $1^{\text {st }} \mathrm{SBM}$ Workshop in (Company, Piquer and Contero, 2004) and (Varley, Martin and Suzuki, 2004).

The remainder of the paper outlines the trends since then, and discusses the direction in which current trends are moving.

Section 3 describes extended vertices, which constitute a problem for many existing systems which use line labelling.
Section 4 describes symmetry, a powerful tool for determining design intent.

Section 5 describes progress towards interpretation of drawings depicting curved objects.

\section{BASELINE}

The two systems we take as our baseline are (Company, Contero, Conesa and Piquer, 2004) for interpreting wireframe drawings and (Varley, Martin and Suzuki, 2004) for interpreting natural line drawings. The conclusions are as follows.

Interpretation of drawings depicting extrusions is straightforward, regardless of the complexity of the extruded face.

Interpretation of wireframe drawings depicting normalons and quasi-normalons is straightforward and fast. Interpretation of natural line drawings depicting normalons depends on the ability of the reconstruction engine to determine what lies around the back of the object. The fact that the object can be deduced to be a normalon is often a useful clue to its structure. The object depicted in Figures 1-3 inclusive is at the limit of what can be interpreted in the domain of normalons.

Interpretation of wireframe drawings depicting non-normalons is slower, since an iterative optimisation process is used to inflate the drawing into 3D. It is also less reliable, since there will always be some doubt about the choice of clues used to construct the object, such as which three sets of parallel lines depict edges aligned with the three orthogonal axes.

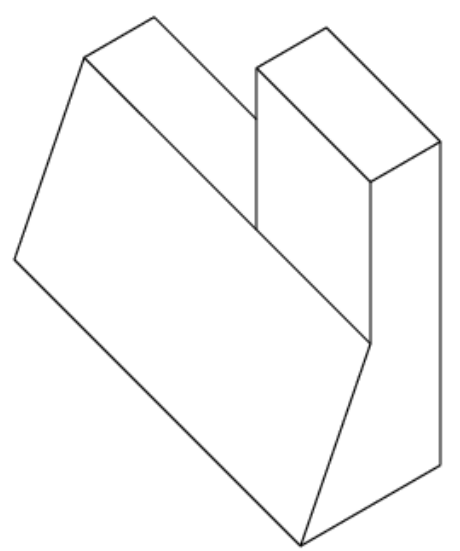

Figure 4: Natural Line Drawing. 
Interpretation of natural line drawings of nonnormalons has met with limited success. Sometimes other clues can be deduced-for example, if all junctions in the drawing are trihedral, it is reasonable to suppose that the depicted object is trihedral too, and this aids the task of the reconstruction engine.

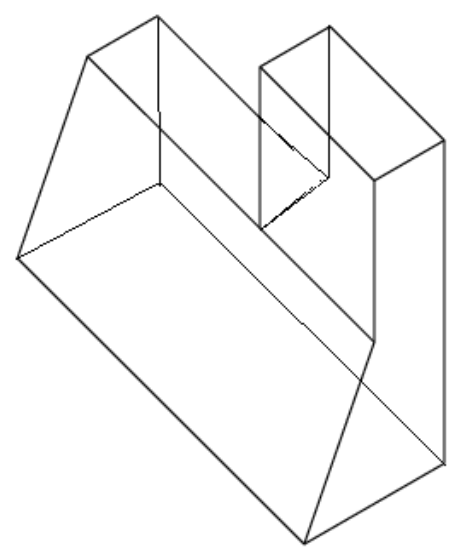

Figure 5: Wireframe Drawing.

However, if even one of the junctions in the drawing is non-trihedral (even implicitly nontrihedral, as in Figure 4), the reconstruction engine has few clues to work with. The object depicted in Figures 4-6 inclusive is at the limit of what can be interpreted in the general-case domain of nontrihedral non-normalons.

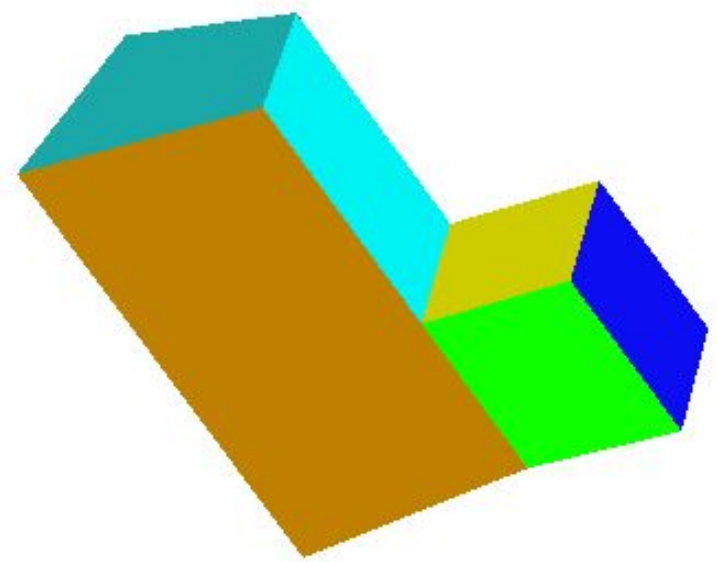

Figure 6: Object.

\section{EXTENDED VERTICES}

This section illustrates extended polyhedral vertices in the domain of natural line drawings. These present a problem for traditional line labelling, and serve to explain why recent systems have used alternative methods of analysing frontal (visible) geometry.

Line labelling as a concept was introduced by (Huffman, 1971) and was first implemented by (Clowes, 1971). Each line in the drawing is labelled as either convex, concave or occluding. By this means, useful clues to the hidden part of the object can be deduced.

Traditional line labelling algorithms treat line labelling as a purely combinatorial constraint satisfaction problem, with 1-node constraints (each junction must have a valid labelling) and 2-node constraints (each line must have the same label throughout its length). The catalogue of valid junction labels for the domain of trihedral polyhedra were determined by (Huffman, 1971). Other catalogues followed, including those for trihedral curved objects (Malik, 1987) and tetrahedral polyhedra (Varley and Martin, 2001).

The first problem with line labelling is conceptual: it cannot be a good idea to ignore the geometry of the drawing at such an early stage of processing.

The second problem with line labelling is practical. Although those known line labelling algorithms which are guaranteed to terminate are in principle $\mathrm{O}\left(\mathrm{e}^{\mathrm{n}}\right)$, in the domain of trihedral polyhedra, practical performance often approaches $\mathrm{O}(\mathrm{n})$ (Parodi et al, 1998). The reason for this is that the junction catalogue for trihedral polyhedra is sparse: there is often only one valid labelling, and line labelling algorithms find it quickly. However, other catalogues are not sparse, drawings of non-trihedral objects often have many valid labellings, and practical performance for these approaches $\mathrm{O}\left(\mathrm{e}^{\mathrm{n}}\right)$.

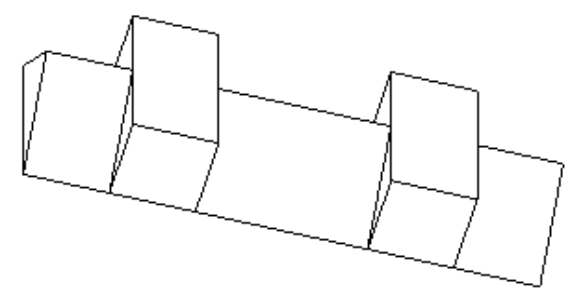

Figure 7: Problem Pentahedral Vertices.

Figures 7, 8 and 9, taken from (Varley 2005), show drawings which catalogue-based labelling cannot label. Even the pentahedral catalogue, 
required for Figure 7, is too large for practical implementation, and there is the further problem in this figure that a T-junction label which in the trihedral domain always indicates an occluding $\mathrm{T}$ junction here corresponds to a genuine vertex.

The hexahedral and heptahedral junction catalogues, are larger still-note that the junction of six lines in Figure 8 implies the presence of a seventh edge in the corresponding vertex of the object, so the heptahedral catalogue would be required. In addition, the hexahedral and higherorder catalogues introduce the problem of two lines which appear in the drawing to cross at a point which is not the termination point of any line-an example of this can also be seen in Figure 8.

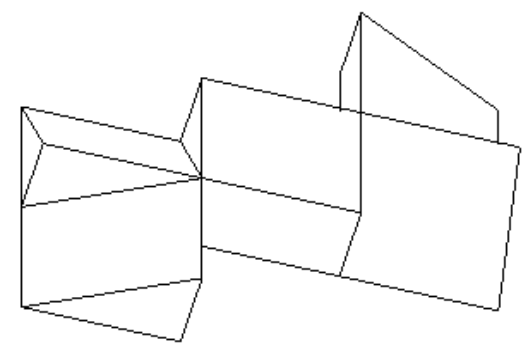

Figure 8: Problem Higher-Valency Vertices.

Figures 7 and 8 could, potentially, depict real engineering objects. Figure 9 does depict a real object, one which can be seen on many computer keyboards. It is safe to say that the junction catalogues required to label this object will not be implemented in the foreseeable future.

Knowing that a problem exists is one thing;

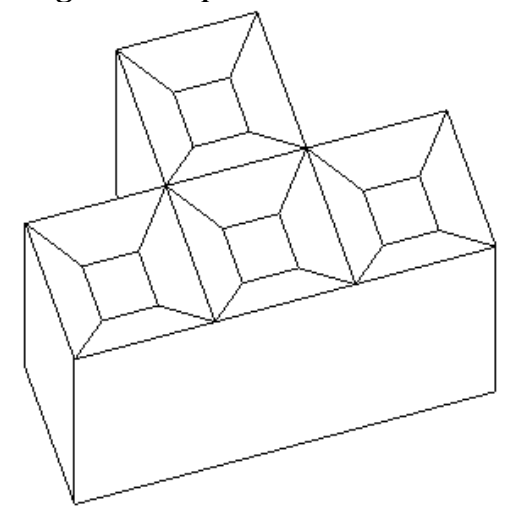

Figure 9: Possible Engineering Object?

finding the solution is another. However, the trend seems to be clearly away from full line labelling. For example, (Ku, Qin and Wright, 2006), who follow tradition in most other respects, make no use of line labelling.

One possibile alternative is to allow user selection at some point in the process. For example, (Kaplan and Cohen, 2006), who use Malik's catalogue for curved objects (Malik, 1987), found it necessary to allow for manual intervention to guide their labelling algorithm. However, this goes against our desire for an approach which requires the user to do nothing more than provide the initial sketch.

Another possibility, which we propose to investigate, is whether partial line labelling (labelling those edges which are in some way obvious, while leaving unlabelled those edges which are uncertain) provides enough useful clues for the reconstruction engine.

Although line labelling as a tool has proved to be a dead end, analysing why line labelling works in the trihedral domain provides geometric insight which remains useful in extended domains even though the technique itself can no longer be recommended.

\section{DESIGN INTENT AND SYMMETRY}

This section discusses various uses of symmetry, especially mirror symmetry, in determining design intent.

The problem of determining design intent is simple to state: what object did the user have in mind when creating the drawing?

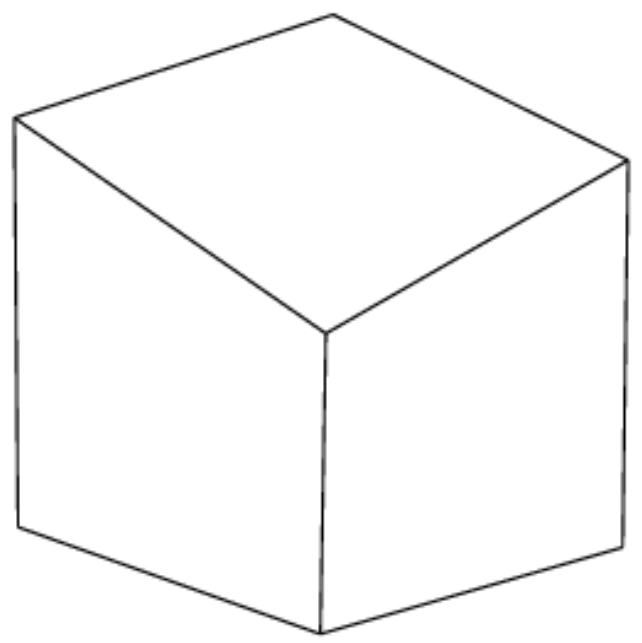

Figure 10: Misplaced Vertex? 
Figure 10 shows a simple example of the design intent problem. Clearly, if the user intended to draw a cube, the central vertex is misplaced. Was this, or was this not, deliberate?

Figure 11 shows a more subtle example, and one which could realistically occur in practice. The height of the central feature is slightly less than the height of the bounding box. Is this, or is this not, deliberate? Depending on which interpretation we choose, we get a different object. If we assume that the difference was deliberate, the central feature is a pocket, and the object is a tray. If we assume that the difference was accidental, the central feature is a through hole, and the object is a ring.

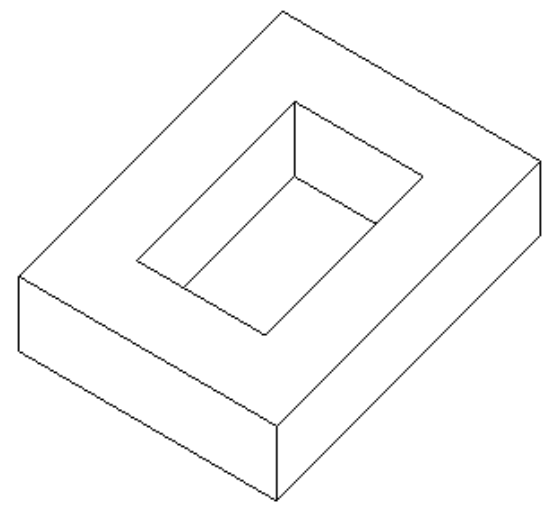

Figure 11: Tray or Ring?

If determining design intent is difficult even for those parts of the object we can see, it is even more difficult for those parts of the object we cannot see. What, for example, is around the back of Figure 12?

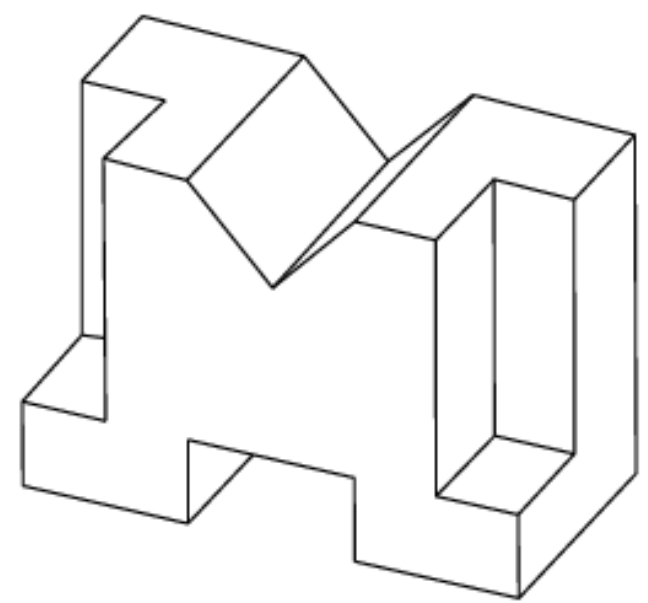

Figure 12: What is the Back?
Since Marill's pioneering work on inflation (Marill, 1991), various clues, both geometrical and perceptual, have been proposed in order to try to capture different aspects of design intent. (Lipson and Shpitalni, 1996) catalogued twelve regularities which could be used for this purpose. However, the problem of determining design intent remains difficult to solve. Even apparently simple tasks such as finding faces-the current state of the art, (Liu and Lee, 2002), uses a genetic algorithm for thisand finding the three main orthogonal axis-see (Masry and Lipson, 2005) for wireframes and (Varley, Martin and Suzuki, 2005) for natural line drawings-are still challenging problems.

Nevertheless, by making assumptions about engineering objects and the ways people see and depict them, it is often possible to reproduce a single object which humans will agree is the correct interpretation of the drawing.

In trying to determine design intent, we believe that we should assume certain regularities whenever it is reasonable to do so. These regularities should be those which are readily perceived, chiefly perpendicularity and symmetry.

Geometrical techniques for identifying and enforcing perpendicularity are well established. (Martin, Varley and Suzuki, 2005) collects several of these.

Enforcing symmetry is also straightforward, but techniques for identifying candidate symmetries and evaluating their merits are still work in progress. Nevertheless, the power of symmetry as a tool is evident. For example, once we have determined that the object depicted in Figure 12 is mirror-symmetric or the object depicted in figure 8 is axis-symmetric, we are close to reconstructing them entirely.

At this stage, it is not even clear at what stage of the process we should attempt to identify candidate symmetries. Clearly, if we have a wireframe already inflated to $3 \mathrm{D}$, identifying candidate symmetries is straightforward. But knowledge of the presence of such a symmetry would be very useful in performing the inflation. So which should be done first, inflation or detection of symmetry? This is a question we propose to investigate. (It should be noted that the same question, the same arguments, and the same uncertain conclusion, also apply to identifying faces in wireframes.)

The answer is likely to depend on the quality of algorithms available for detection of symmetry in 2D wireframes. This is not purely a graph isomorphism problem-the geometry of the wireframe must also be considered-so there is considerable room for improvement in this area. 
Identification of candidate symmetries in 2D natural line drawings is even more of a problem. This is most difficult when different topology is visible on the "near" and "far" sides of the mirror plane, as in Figure 12, but even when the mirror plane bisects the visible topology, finding it is not always straightforward. For example, we know of no algorithm which detects only the "obvious" (to a human) topological mirror symmetry in Figure 13 (and we should welcome a contribution from anyone who does!).

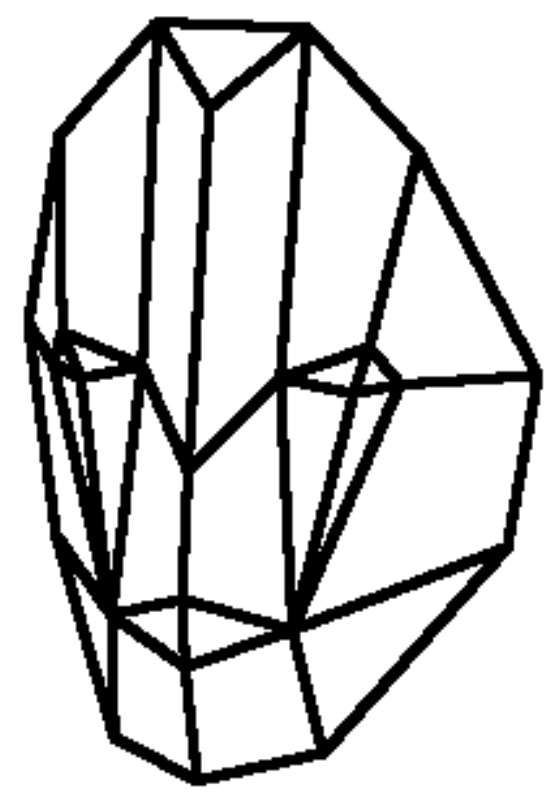

Figure 13: Where is the Symmetry? (Takahashi, 2004)

\section{CURVED OBJECTS}

This section discusses various systems which allow curved objects to be sketched.

In general, the more knowledge that can be built in to the interpretation system, the better that system will perform. For example, the method of Constellation Models (Yang, Sharon and van de Panne, 2005) (Sharon and van de Panne, 2006) is generally successful in interpreting sketches of five specific classes of object: face, flower, sailboat, aeroplane or humanoid character. Each sketch is assumed to be of an object of one of these classes. It is allocated to the class which it matches best, and analysed using domain-specific knowledge relating to that class. Even with only five classes, the occasional sketch is allocated to the wrong class, and it seems inevitable that this misallocation will increase as further classes are added. Additionally, it is impossible to sketch any object not belonging to one of these five classes.

The approach of (Takahashi, 2004) and (Varley, Takahashi, Mitani and Suzuki, 2004), although apparently more general, is in practice equally limited. A sketch is interpreted by means of a polyhedral template, either prepared in advance (Takahashi, 2004) or created from, and topologically equivalent to, the curved lines drawn by the user (Varley, Takahashi, Mitani and Suzuki, 2004). In the former case, the corresponding polyhedron is specified when creating the template. In the latter case, the corresponding polyhedron, which is assumed to contain a plane of mirror symmetry, is created using the methods described above (sections 2 and 4). In both cases, the 3D polyhedron is then recurved to match the user's original drawing using Loop subdivision (Loop, 1987). The assumption of mirror symmetry is necessary in order to allow the hidden part of the object to be curved. There are a number of problems with this approach, not least that, as seen in section 4, there is at present no reliable algorithm for detecting 3D planes of mirror symmetry in 2D natural line drawings.

The choice of Loop subdivision may not be ideal-it is possible that other subdivision algorithms would produce better results, and alternatives such as the FIN algorithm (Gross, 2005) are worth investigating. In considering such algorithms, we must note a problem which must be avoided: careless triangulation can lose the mirror symmetry which we have gone to so much trouble to identify.

The approach of (Kara and Shimada, 2006) deforms a single polyhedral template in response to curved strokes entered by the user. As with (Takahashi, 2004), the template must be created separately, but can be re-used for similar objects. The assumption made in deforming the template is physical rather than geometric: they imagine that the faces of the template are thin membranes on which pressure forces are exerted. Perhaps because of this similarity to real-world objects, the results of this process have an attractive appearance.

The approach of (Kaplan and Cohen, 2006) creates a $2 \frac{1}{2} \mathrm{D}$ model. This demonstrates the limits of what can be achieved without any assumptions or templates. No attempt is (or can be) made to deduce the hidden part of the object.

The quality of output achieved by the constraintbased reconstruction used by (Kaplan and Cohen, 2006) is very high, but this comes at a price: 
rendering their final curved object takes minutes rather than seconds or (ideally) fractions of seconds.

\section{ACKNOWLEDGEMENTS}

The support of the Japan Society for the Promotion of Science (Fellowship no P03717) and the Ramon y Cajal Scholarship Programme is acknowledged with gratitude.

\section{REFERENCES}

Clowes, M., 1971. On Seeing Things. Artificial Intelligence 2, 79-116.

Company, P., Contero, M., Conesa, J., Piquer, A., 2004. An Optimization-Based Reconstruction Engine for 3D Modelling by Sketching, Computers and Graphics 28(6), 955-979.

Company, P., Piquer, A., Contero, M., On the Evolution of Geometrical Reconstruction as a Core Technology to Sketch-Based Modelling, In ed. J. F. Hughes and J. A. Jorge, Sketch-Based Interfaces and Modelling, Eurographics Symposium Proceedings, 97-106. Eurographics Press.

Gross, N, 2005. The FIN Algorithm-Using Subdivision Methods for Computer Aided Design and Manufacturing. Computer Graphics and Imaging, 4953.

Huffman, D., 1971. Impossible Objects as Nonsense Sentences. Machine Intelligence 6, 295-323.

Kaplan, M, Cohen, E, 2006. Producing Models From Drawings of Curved Surfaces, in ed. T. Stahovich and M. Costa Sousa, Eurographics Workshop on SketchBased Interfaces and Modelling, 51-58.

Kara, L.B., Shimada, T.,2006.Construction and Modification of 3D Geometry Using a Sketch-Based Interface, in ed. T. Stahovich and M. Costa Sousa, Eurographics Workshop on Sketch-Based Interfaces and Modelling, 59-66.

Ku, D.C., Qin, S.F., Wright, D.K., 2006. A Sketching Interface for 3D Modelling of Polyhedrons, in ed. T. Stahovich and M. Costa Sousa, Eurographics Workshop on Sketch-Based Interfaces and Modelling, 83-90.

Lipson H. and Shpitalni M. 1996. Optimization-Based Reconstruction of a 3D Object from a Single Freehand Line Drawing. Computer-Aided Design. 28(8), 651663.

Liu J. and Lee Y.T. 2002. Identifying Faces in a 2D Line Drawing Representing a Manifold Object, IEEE Transactions on Pattern Analysis and Machine Intelligence, 24(12), 1579-1593.

Loop, C.T., 1987. Smooth Subdivision Surfaces Based on Triangles. Department of Mathematics, University of Utah.
Malik, J., 1987. Interpreting Line Drawings of Curved Objects. International Journal of Computer Vision, 73-103.

Marill, T. 1991. Emulating the Human Interpretation of Line-Drawings as Three-Dimensional Objects. International Journal of Computer Vision 6(2), 147161.

Martin, R.R., Varley, P., Suzuki, H, 2005. Perpendicularity as a Key to Interpreting Line Drawings of Engineering Objects, in Proc. Digital Engineering Workshop: 5th Japan-Korea CAD/CAM Workshop, 115-120.

Masry M., Lipson H. 2005. A Sketch-Based Interface for Iterative Design and Analysis of 3D Objects. in ed. $J$. F. Hughes and J. A. Jorge, Sketch-Based Interfaces and Modelling, Eurographics Symposium Proceedings,109-118.

Parodi, P., Lancewicki, R., Vijh, A., Tsotsos, J. K., 1998. Empirically-Derived Estimates of the Complexity of Labeling Line Drawings of Polyhedral Scenes. Artificial Intelligence 105, 47-75.

Sharon, D, van de Panne, M, 2006. Constellation Models for Sketch Recognition, in ed. T. Stahovich and $M$. Costa Sousa, Eurographics Workshop on SketchBased Interfaces and Modelling, 19-26.

Takahashi, Y., 2004. Constructing 3D from Sketch Portraits according to Prepared Templates, MSc Thesis, The University of Tokyo. In Japanese.

Varley, P., 2005. Extended Vertices: A Problem for Line Labelling, in Proc. Digital Engineering Workshop: 5th Japan-Korea CAD/CAM Workshop, 106-114.

Varley, P.A.C., Martin, R.R., 2001. The Junction Catalogue for Labelling Line Drawings of Polyhedra with Tetrahedral Vertices, International Journal of Shape Modelling 7(1), 23-44.

Varley, P.A.C., Martin, R.R., Suzuki, H, 2004. Can Machines Interpret Line Drawings? In ed. J. F. Hughes and J. A. Jorge, Sketch-Based Interfaces and Modelling, Eurographics Symposium Proceedings, 107-116.

Varley, P.A.C., Martin, R.R., Suzuki, H, 2005. Frontal Geometry from Sketches of Engineering Objects: Is Line Labelling Necessary? Computer Aided Design 37 (12), 1285-1307.

Varley, P.A.C., Takahashi, Y., Mitani, J., Suzuki, H., 2004. A Two-Stage Approach for Interpreting Line Drawings of Curved Objects, in ed. J. F. Hughes and J. A. Jorge, Sketch-Based Interfaces and Modelling, Eurographics Symposium Proceedings, 117-126.

Yang, C., Sharon, D, van de Panne, M, 2005. SketchBased Modelling of Parameterized Objects, in ed. T. Igarashi and J.A. Jorge, Eurographics Workshop on Sketch-Based Interfaces and Modelling, 63-72. 